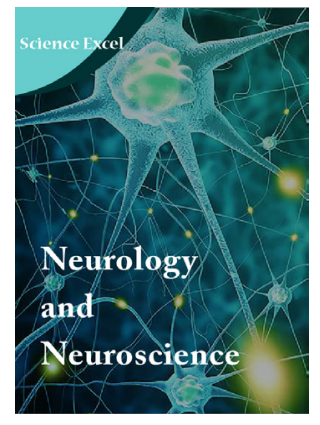

\section{Correspondence}

\section{Haley Tucker}

University of Texas at Austin, Austin, USA

Tel. (+1) 512-232-0859

Fax. (+1) 512-475-7707

E-mail: haleytucker@austin.utexas.edu

- Received Date: 06 Sep 2019;

- Accepted Date: 19 Sep 2019.

- Publication Date: 23 Sep 2019.

\section{Keywords:}

malaria; innate immune response; adaptive immune response; vaccine

\section{Copyright}

(c) 2019 Science Excel. This is an openaccess article distributed under the terms of the Creative Commons Attribution 4.0 International license.

\title{
Malaria: pathology, immune response, and vaccine design: A mini review
}

\author{
Henry S Pan ${ }^{1}$ and Haley 0 Tucker ${ }^{2 *}$ \\ 'Department of Chemical Engineering, University of Texas at Austin, Austin, Texas, USA \\ ${ }^{2}$ Institute for Cellular and Molecular Biology and Department of Molecular Biosciences, University of Texas at Austin, Austin, \\ Texas, USA
}

\begin{abstract}
Malaria is a mosquito-borne disease for which nearly half the world's population is at risk, with most cases occurring in sub-Saharan Africa. A significant number of deaths of children under the age of 5 have resulted from cerebral malaria. Ongoing treatments, such as quinine, have existed for over 300 years, and recent treatments have incorporated artesunate. However, effective treatments still are necessary in order to prevent malaria and the resulting symptomology. Ongoing work has been conducted to investigate the underlying mechanism of the disease and how the body addresses it through innate and adaptive immunity. The aim of this review is to explore the current understanding of the immune response to malaria's progression in the liver, bloodstream, and brain as well as to assess recent developments in engineering an efficacious vaccine.
\end{abstract}

\section{Introduction}

Malaria is a parasitic-driven disease, with over 200 million new cases and 400,000 deaths annually occurring [1]. African regions are the most affected, accounting for over $90 \%$ of the cases [1]. More than $60 \%$ of deaths comprise of children under the age of 5-an especially vulnerable population, due to their underdeveloped immune response [1]. The parasite Plasmodium vivax is the most geographically widespread species of malaria, along with 4 other Plasmodium strains known to cause the disease in humans [1]. Despite decades of research to reduce the mortality rate, especially in children, there is currently only one candidate vaccine in Phase III clinical trials that has shown a protective effect in young children [2].

\section{Literature Review}

\section{Pathology}

The malaria infection begins when an infected mosquito bites a human and transfers the parasite sporozoites through its saliva. The sporozoites travel through the bloodstream until reaching the liver, where they infect a few hepatocytes and asexually reproduce (Figure 1). Merozoites are released after completing the first round of replication in the hepatocytes, from which they disperse back into the bloodstream to infect red blood cells (RBCs). The cycle of malaria then repeats, with replication of merozoites in the infected RBCs, waves of synchronized schizogeny, and infection of new RBCs.

The disease becomes especially lethal once the merozoites cross the blood-brain barrier (BBB) to elicit an immune response within the cerebrum, triggering a cytokine storm [3]. This leads to cerebral malaria $(\mathrm{CM})$, which causes $15 \%$ to $20 \%$ of case fatalities in children [4]. Current work is underway to investigate the mechanisms governing this fatal outcome in CM. These include the impact of the proinflammatory response and the adaptive immune response on CM pathology [5]. Several studies observing the proinflammatory response from the peripheral cytokines of children presenting with CM examined which cytokines correlated with CM symptomology. In one study, children presenting with $\mathrm{CM}$ and in comatose, produced no single cytokine that could predict brain swelling, despite elevation of specific cytokines correlating with CM and high parasiteamia [4]. In another study with children presenting with $\mathrm{CM}$, it was found that levels of angiopoietin-1 (Ang-1) - a growth factor critical for endothelial cell function through its interaction with Tie-2-were associated with disease severity [5].

Subsequently, it was observed that therapeutic Ang-1 administered with artesunate improved vascular integrity and survival in mouse models of Experimental Cerebral Malaria (ECM) [6]. Further studies of ECM in mouse models compared the separate roles that adhesion of parasite-specific $\mathrm{CD}^{+}$ $\mathrm{T}$ cells played on luminal brain vascular endothelial cells (ECs) as compared to the adhesion of infected red blood cells (iRBCs) [7]. Their results suggested that anti-adhesion molecule (e.g. LFA-1/VLA-4) therapy might be efficacious as a potential avenue for preventing cerebral malaria.

\section{Innate immune response}

The innate immune system provides both receptors that detect the onset of malaria and an early protective immune response. While innate immunity can provide initial protection before the adaptive response takes place, it can also become overreactive, causing severe clinical manifestations of the disease. 


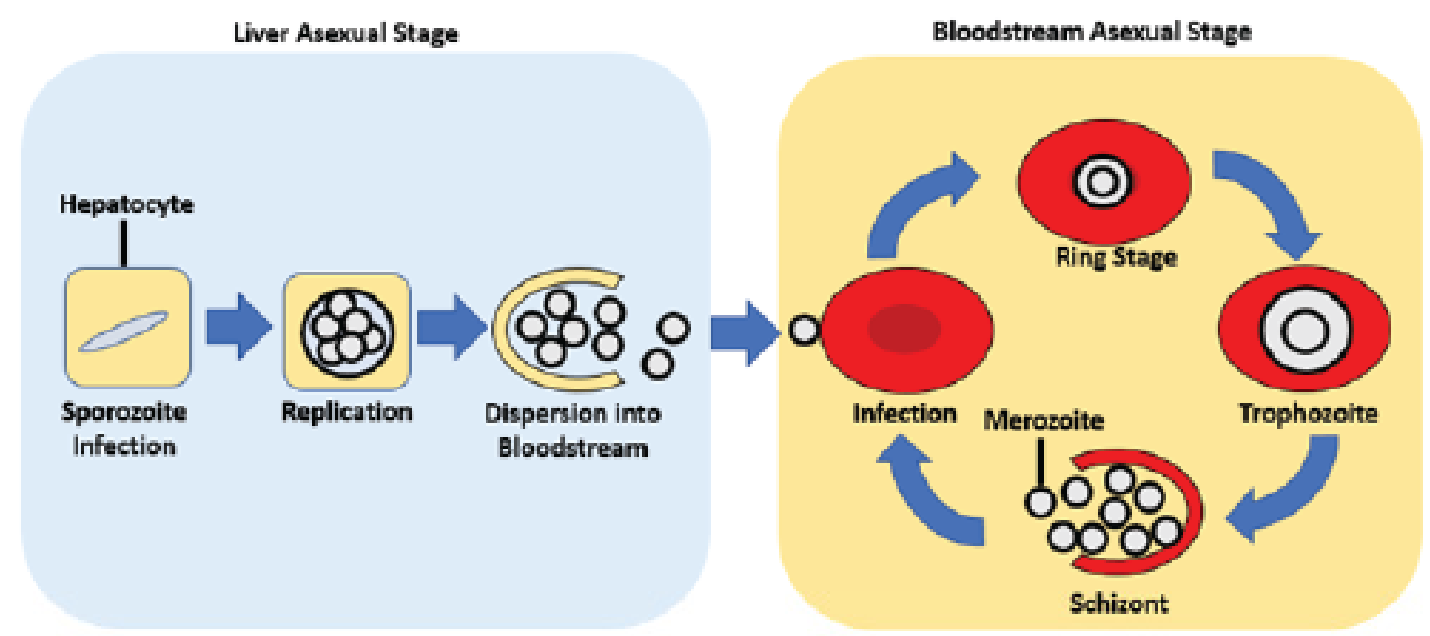

Figure 1. A schematic of liver (left) and bloodstream (right) stages of malaria parasite infection and replication.

\section{Interferon- $\gamma$}

During the malarial innate immune response, interferon- $\gamma$ (IFN- $\gamma$ ) plays an important role because of its anti-parasitic functions. IFN- $\gamma$ is secreted by $\mathrm{T}$ helper $1\left(\mathrm{~T}_{\mathrm{H}} 1\right)$ cells, $\mathrm{CD}^{+}$cytotoxic $\mathrm{T}$ cells, and natural killer (NK) cells. This cytokine is primarily used to block viral replication. It is also involved in triggering additional macrophage and NK cell activation. However, it can also upregulate Major HistoCompatibility complex (MHC) class I display on infected cells, to promote $\mathrm{CD}^{+} \mathrm{T}$ cell clearage of parasites within infected RBCs and hepatocytes.

Typically, when macrophages produce IL-18 to act in synergy with IL-12, NK cells are stimulated initially to produce IFN- $\gamma$ prior to maturation of $\mathrm{CD}^{+} \mathrm{T}$ cells and $\mathrm{T}_{\mathrm{H}} 1$ cells [8]. In malaria, this early IFN- $\gamma$ production from NK cells helps to promote macrophage activation prior to the onset of the adaptive immune response, which leads to IFN- $\gamma$ production in $\mathrm{T}_{\mathrm{H}} 1$ cells. In one study in which mice were subjected to both live and dead malaria parasites, activation of IFN- $\gamma$ production in NK cells was confirmed to be IL-12, but not IL-18, dependent [8]. Also, these investigators found that humans reacting to infected RBCs had heterogeneous levels of NK cell/IFN- $\gamma$ production in responses to malaria; i.e. some but not all patients produced large amounts of IFN- $\gamma$. These results led to two opposing hypotheses: that rapid IFN- $\gamma$ production was either promoting an enhanced ability to control malaria, or that the responding patients were predisposed to severe malarial infection due to cytokine overproduction [9].

\section{TLR9-MyD88 signaling}

The Toll-Like Receptor (TLR) 9 and the MyD88 adaptor signaling pathway is critical for the activation of dendritic cells (DC), which in turn, help to bridge innate and adaptive immunity. It was originally thought that TLR9 activation was caused by hemozoin (HZ), a parasite detoxification product of $\mathrm{RBC}$ heme molecules widely used as a biomarker to assess malarial infection severity [10].

This hypothesis was followed up by in vitro and in vivo mouse model studies aimed at testing both HZ purified from P. falciparum, as well as a synthetic HZ, that was free from possible contaminants. Wild type (WT) along with TLR2 $2^{-/} / \mathrm{TLR}^{-1 /} / \mathrm{TLR}{ }^{-/}$triple knockout mice could be activated by HZ although neither CD40 nor CD86 were upregulated (both these $\mathrm{T}$ cell coreceptors are typically upregulated by proinflammatory cytokines). In contrast, TLR9 $9^{-/-}$mice, in which CD40 and CD86 were expressed at normal levels, were not activated. These results indicated that $\mathrm{HZ}$ activation was TLR9 dependent [11].

A study conducted by Wu et al. [12] then found that protein-DNA complexes were the sole component in activating the TLR9-MyD88 signaling pathway and thus, concluded that HZ is not a TLR9 ligand [12]. Follow-up studies concluded that while $\mathrm{HZ}$ was immunologically inert, it still played a role in TLR9 activation by presenting malaria DNA to TLR9 [13].

\section{The adaptive immune response}

In the adaptive immune response to malaria, much of the interest has focused on the role of B cells, since antibodies provide many effector functions that both prevent invasion and promote parasite clearance. However, some of the focus also has shifted recently toward $\mathrm{T}$ cells and their role in both eliminating infected cells and providing cytokines to help with B cell function.

\section{B cells}

$\mathrm{B}$ cells are a central part of the adaptive immune response to malaria because of their role in producing parasite-specific antibodies (Abs). These Abs promote infection clearance during the acute response as well as develop enduring humoral memory after the initial infection. The understanding of malaria-specific Abs is especially important, because they are thought to be the cause of naturally acquired humoral immunity in patients who are capable of clearing the infection on first encounter. It has been demonstrated that sera transferred from malaria-immune adults to children could protect them from clinical disease, further reinforcing the need to better understand natural $\mathrm{Ab}$ function [14]

Over the course of an acute malarial infection, somatic hypermutation within $\mathrm{Ab}$ variable regions increase mutation loads onto both $\mathrm{B}$ cell antigen receptors and Abs to enhance affinity. Observing this accumulation of mutations was previously challenging, because amplification errors in sequencing has made discerning real but subtle mutations difficult. With the introduction of molecular barcoding technology, recent studies are now able to better track $\mathrm{Ab}$ repertoire features during various phenomena [15], including response to disease or to vaccination [16]. Observing Ab repertoire changes with this level of accuracy offers insight as to how mutation load changes within different $\mathrm{Ab}$ isotypes that modulate an individual's adaptive immune response [17]. 
Another question that the field is currently studying is how age affects the $\mathrm{B}$ cell response, especially in the context of vaccine development for infants and toddlers. During an individual's lifetime, antigen exposure to the general environment allows for the immunorepertoire to build an adaptive immune response by accumulating effective memory $\mathrm{T}$ and $\mathrm{B}$ cells. Prior to this diversification, infants and toddlers are extremely vulnerable to infection. They may respond differently to vaccines because they have not had sufficient time to diversify their immunorepertoire [18]. It was shown recently that $\operatorname{IgM}^{+}$memory B cells seem to promote early parasite clearance as compared to $\operatorname{IgM}^{-}$memory B cells [19]. This indicated that $\operatorname{IgM}^{+} B$ cell formation following vaccination can serve as a metric for the efficacy of a vaccine formulation in younger patients.

\section{CD4 ${ }^{+} T$ cells}

Because B cells can respond to pre-erythrocytic stages of malaria, it is inferred that helper $\left(\mathrm{CD} 4^{+}\right) \mathrm{T}$ cells must function in helping $\mathrm{B}$ cells secrete Abs and become memory B cells, since they are necessary for the activation of naïve $B$ cells. $\mathrm{T}_{\mathrm{H}} 1$ cells play an important role in secreting IFN- $\gamma$ which helps in priming the innate immune response and maintaining long-term protection from malaria [20]. T follicular helper $\left(\mathrm{T}_{\mathrm{FH}}\right)$ cells are also known to play an important role in germinal center B cell development [8], although far less is known about their specific contribution to a malarial response.

Also, there is an ongoing interest in better understanding the transcriptional changes that naïve $\mathrm{T}$ cells undergo when differentiation into different $\mathrm{CD} 4^{+} \mathrm{T}$ cell subsets. In a recent study [19], single cell RNA sequencing of differentiating $\mathrm{CD} 4^{+} \mathrm{T}$ cells revealed the transcriptomic changes naïve $\mathrm{T} \mathrm{CD} 4^{+} \mathrm{T}$ cell experienced when bifurcating into either $\mathrm{T}_{\mathrm{H}} 1$ or $\mathrm{T}_{\mathrm{FH}}$ cell types.

By sequencing at different time points during an acute malaria infection in WT mice, de Silva et al. [20] established a "pipeline" which visually displayed the gene trajectory of the $\mathrm{CD} 4^{+} \mathrm{T}$ cells and determine specific transcription factors required for differentiation fate. These included $T c f 7$ and Bcl6 for $\mathrm{T}_{\mathrm{FH}}$ fate, along with Cxcr6 and $I d 2$ for $\mathrm{T}_{\mathrm{H}} 1$ fate. $T c f 7$ is known to be instrumental in $\mathrm{T}_{\mathrm{FH}}$ differentiation, and $I d 2$ is an agonist of Tcf7 involved in the $\mathrm{TH} 1$ response [21].

With further analysis of the $\mathrm{T}$ cell transcriptome during an acute malaria infection, the underlying genetic mechanisms that govern the responding $\mathrm{T}$ cell response can help inform the molecular relationships of $\mathrm{T}$ cell fate.

\section{Vaccine development}

Currently, efforts are underway to generate two different types of vaccines: attenuated live parasites and recombinant protein-based vaccines. Currently, RTS,S/ASO1 is the most developed recombinant protein-based vaccine that has shown efficacy in clinical phase III trials [2]. Attenuated malaria parasites also have shown moderate success, demonstrating that they are safe in humans [22]. The current aim of these vaccines is to induce patients to develop Abs specific to either sporozoites or merozoites (Figure 1).

\section{RTS,S/ASO1}

In a phase III trial conducted on African infants, this vaccine was shown to reduce clinical and severe malaria by $50 \%$. After the administering of the third dose of the vaccine, $99.7 \%$ of children developed anticircumsporozoite Abs [23]. During the trial, three monthly doses were given followed by a month 20 booster shot. The participants were randomized to include RTS,S/ASO1 in either all of the shots, all except the booster, or all comparator vaccines. It was observed that over a time course following each injection regardless of vaccine schedule, the RTS,S/ASO1 efficacy decreased substantially. Additionally, primary vaccination in toddlers showed better efficacy than primary vaccination in infants [2].

\section{Live attenuated vaccines}

By knocking out genes necessary for productive replication of the sporozoites or irradiating the sporozoites, it is possible to induce protective immunity without risking the progression of malaria infection using live malaria parasites [8]. The benefit of live attenuated vaccines is that they offer sterile protection; ie, no malaria parasites reach the blood phase of their life cycle. In a clinical trial conducted on a Plasmodium falciparum sporozoite (PfSPZ) vaccine, human volunteers were immunized by mosquitoes that contained irradiated sporozoites in their salivary glands. The efficacy was not high, as indicated by low $\mathrm{T}$ cell and $\mathrm{Ab}$ responses against PfSPZ. However, the problem likely resulted from the method employed for immunization [22]. A recent study using PfSPZ was then able to provide sterile protection in roughly $50 \%$ of subjects, indicating the progress of these vaccine protocols [22,24].

\section{Conclusion}

While progress has been made toward developing a successful vaccine to malaria, there are still many questions that need to be answered to develop measurements of success in different vaccines. The mechanisms governing the innate and adaptive response still require further exploration, especially for infants and toddlers [17]. The advent of immunorepertoire sequencing particularly can help with this understanding by assessing transcriptome and genetic changes that occur during the effector and memory responses. Such understanding can guide recombinant protein-based vaccine design in the context of testing different antigenic targets and adjuvants. Vaccines that can induce a strong $\operatorname{IgM}^{+}$memory B cell response are likely to be the most efficacious.

\section{Acknowledgements}

We thank members of the Tucker and Lauren Webb laboratories for discussions and reading of the manuscript. Support for this work was provided by NIH Grant R01CA31534, Cancer Prevention Research Institute of Texas (CPRIT) Grants RP100612, RP120348; and the Marie Betzner Morrow Centennial Endowment to H.O.T.

\section{Author contributions}

HSP wrote the manuscript. HOT edited the manuscript.

\section{Competing financial interests}

The authors declare no competing financial interest.

\section{References}

1. WHO | World Malaria Report 2016. WHO (2017).

2. RTS,S Clinical Trials Partnership. Efficacy and safety of RTS,S/AS01 malaria vaccine with or without a booster dose in infants and children in Africa: final results of a phase 3, individually randomised, controlled trial. Lancet. 2015; 386(9988):31-45.

3. Cowman AF, Crabb BS. Invasion of red blood cells by malaria parasites. Cell. 2006; 124(4):755-766.

4. Seydel KB, Kampondeni SD, Valim C, et al. Brain swelling and death in children with cerebral malaria. N Engl J Med. 2015; 372:1126-1137.

5. Nishanth G, Schlüter D. Blood-brain barrier in cerebral malaria: Pathogenesis and therapeutic intervention. Trends Parasitol. 2019; 35(7):516-528.

6. Higgins SJ, Purcell LA, Silver KL, et al. Dysregulation of angiopoietin-1 plays a mechanistic role in the pathogenesis of cerebral malaria. Sci Transl Med. 2016; 8(358):358ra128.

7. Swanson PA 2nd, Hart GT, Russo MV, et al. CD8+ T cells induce fatal brainstem pathology during cerebral malaria via luminal antigen-specific engagement of brain vasculature. PLoS Pathog. 2016; 12(12):e1006022.

8. Murphy K, Weaver C. Janeway's Immunobiology. Garland Science. New York, USA. 2016.

9. Artavanis-Tsakonas K, Riley EM. Innate immune response to malaria: rapid induction of IFN-gamma from human NK cells by live Plasmodium falciparuminfected erythrocytes. J Immunol. 2002; 169(6):2956-63. 
10. Nguyen PH, Day N, Pram TD, et al. Intraleucocytic malaria pigment and prognosis in severe malaria. Trans R Soc Trop Med Hyg. 1995; 89(2):200-4.

11. Coban C, Ishii KJ, Kawai T, et al. Toll-like receptor 9 mediates innate immune activation by the malaria pigment hemozoin. J Exp Med. 2005; 201(1):19-25.

12. Wu X, Gowda NM, Kumar S, et al. Protein-DNA complex is the exclusive malaria parasite component that activates dendritic cells and triggers innate immune responses. J Immunol. 2010;184(8):4338-48.

13. Parroche $P$, Lauw FN, Goutagny $N$, et al. Malaria hemozoin is immunologically inert but radically enhances innate responses by presenting malaria DNA to Toll-like receptor 9. Proc Natl Acad Sci U S A. 2007; 104(6):1919-24.

14. Sabchareon A, Burnouf $T$, Ouattara $D$, et al. Parasitologic and clinical human response to immunoglobulin administration in falciparum malaria. Am JTrop Med Hyg. 1991; 45(3):297-308.

15. Georgiou G, Ippolito GC, Beausang J, et al. The promise and challenge of high throughput sequencing of the antibody repertoire. Nat Biotechnol. 2014; 32(2):158-68.

16. Vollmers C1, Sit RV, Weinstein JA, et al. Genetic measurement of memory B-cell recall using antibody repertoire sequencing. Proc Natl Acad Sci U S A. 2013; 110(33):13463-8.

17. Wendel $\mathrm{BS}, \mathrm{He} C, \mathrm{Qu} \mathrm{M}$, et al. Accurate immune repertoire sequencing reveals malaria infection driven antibody lineage diversification in young children. Nat
Commun. 2017; 8(1):531

18. PrabhuDas M1, Adkins B, Gans $\mathrm{H}$, et al. Challenges in infant immunity: implications for responses to infection and vaccines. Nat Immunol. 2011; 12(3):189-94.

19. Stone SL, Lund FE. IgM Memory Cells: First responders in malaria. Immunity. 201; 45(2):235-7.

20. da Silva HB, de Salles EM, Panatieri RH, et al. IFN- $\gamma$-induced priming maintains long-term strain-transcending immunity against blood-stage Plasmodium chabaudi malaria. J Immunol. 2013; 191(10):5160-9.

21. Lönnberg T, Svensson V, James KR, et al. Single-cell RNA-seq and computational analysis using temporal mixture modelling resolves Th1/Tfh fate bifurcation in malaria. Sci Immunol.2017; 2(9).

22. Epstein JE, Tewari $\mathrm{K}$, Lyke $\mathrm{KE}$, et al. Live attenuated malaria vaccine designed to protect through hepatic CD8囚T cell immunity. Science. 2011; 334(6055):475-80.

23. Agnandji ST, Lell, B Fernandes JF, et al. A phase 3 trial of RTS,S/AS01 malaria vaccine in African infants. N Engl J Med. 2012; 367(24):2284-95.

24. Lyke KE, Ishizuka AS2, Berry AA, et al. Attenuated PfSPZ Vaccine induces straintranscending $T$ cells and durable protection against heterologous controlled human malaria infection. Proc Natl Acad Sci U S A. 2017; 114(10):2711-2716. 